\title{
Long evolutionary history of an emerging fungal pathogen of diverse tree species in eastern Asia, Australia, and the Pacific Islands
}

\author{
Olga Kozhar ${ }^{1}$, Mee-Sook Kim² ${ }^{2}$, Jorge Ibarra Caballero ${ }^{1}$, Ned Klopfenstein ${ }^{3}$, Phil Cannon ${ }^{4}$, \\ and Jane Stewart ${ }^{1}$ \\ ${ }^{1}$ Colorado State University \\ ${ }^{2}$ United States Department of Agriculture Forest Service Pacific Northwest Region \\ ${ }^{3}$ United States Department of Agriculture Forest Service Rocky Mountain Research Station \\ ${ }^{4}$ United States Department of Agriculture Forest Service Forest Health Protection
}

December 29, 2021

\begin{abstract}
Emerging plant pathogens have been increasing exponentially over the last century. To address this issue, it is critical to determine whether these pathogens are native to ecosystems or have been recently introduced. Understanding the ecological and evolutionary processes fostering emergence can help to manage their spread and predict epidemics/epiphytotics. Using restriction site-associated DNA sequencing data, we studied genetic relationships, pathways of spread, and evolutionary history of Phellinus noxius, an emerging root-rotting fungus of unknown origin, in eastern Asia, Australia, and the Pacific Islands. We analyzed patterns of genetic variation using Bayesian inference, maximum likelihood phylogeny, populations splits and mixtures measuring correlations in allele frequencies and genetic drift, and finally applied coalescent based theory using Approximate Bayesian computation (ABC) with supervised machine learning. Population structure analyses revealed five genetic groups with signatures of complex recent and ancient migration histories. The most probable scenario of ancient pathogen spread is movement from ghost population to Malaysia and the Pacific Islands, with subsequent spread to Taiwan and Australia. Furthermore, ABC analyses indicate that $P$. noxius spread occurred thousands of generations ago, contradicting previous assumptions that this pathogen was recently introduced to multiple geographic regions. Our results suggest that recent emergence of $P$. noxius in eastern Asia, Australia, and the Pacific Islands is likely driven by anthropogenic and natural disturbances, such as deforestation, land-use change, severe weather events, and/or introduction of exotic plants. This study provides a novel example of applying genome-wide allele frequency data to unravel dynamics of pathogen emergence under changing ecosystem conditions.
\end{abstract}

\section{Hosted file}

Phellinus_main_text_revision_final.docx available at https://authorea.com/users/426415/ articles/551031-long-evolutionary-history-of-an-emerging-fungal-pathogen-of-diversetree-species-in-eastern-asia-australia-and-the-pacific-islands 
a
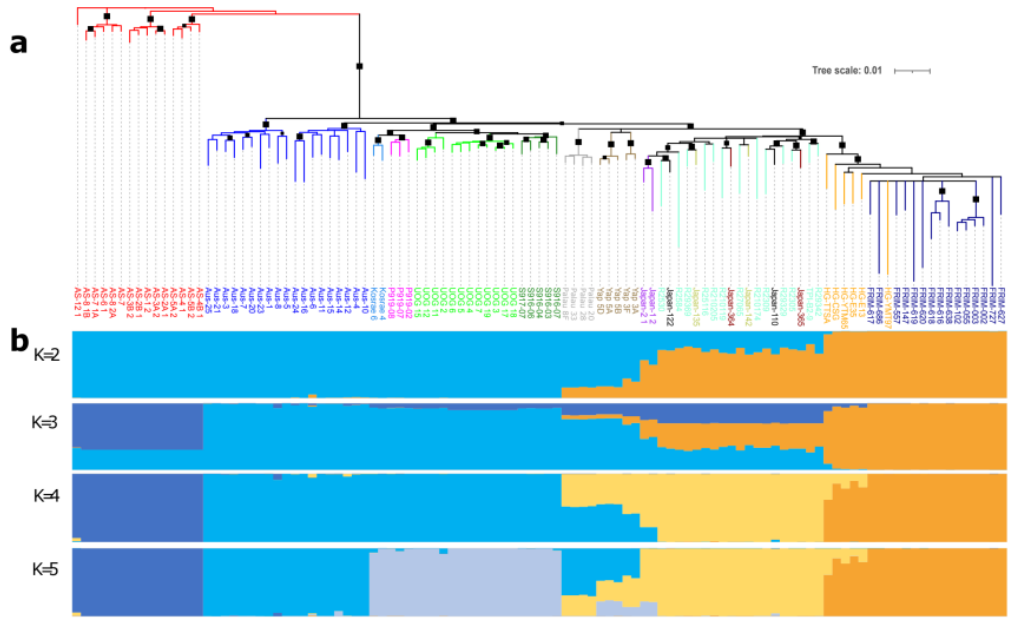

C

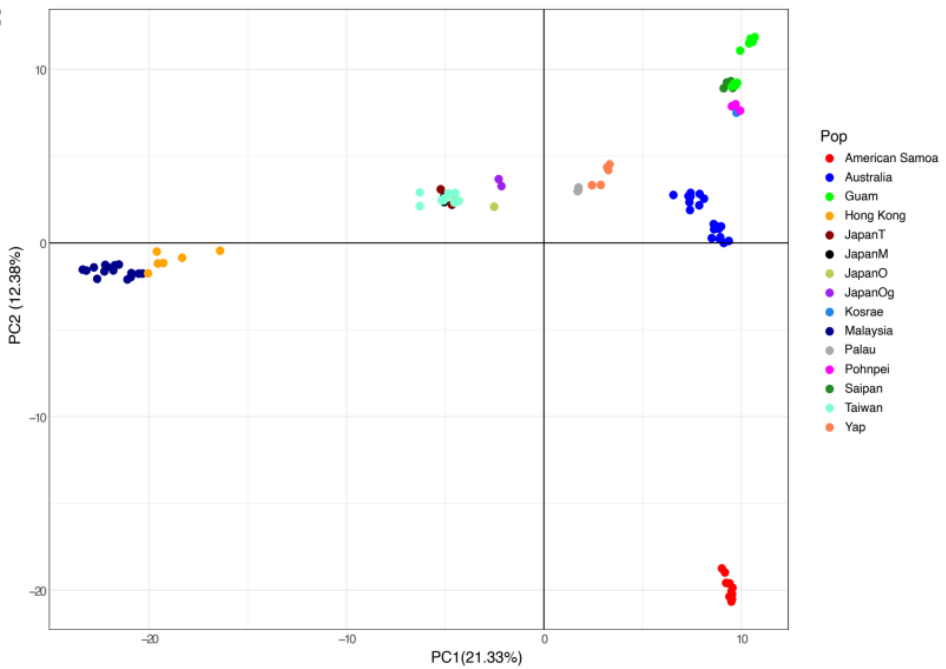

Figure 1. Maximum likelihood phylogenetic tree (a), Structure Bayesian clustering (b), and

Principal component analysis (PCA) (c) of Phellinus noxius populations from 15 locations.

(a) Different colors represent samples from different locations reflected in PCA legend (c).

The branches with $\geq 95 \%$ likelihood support are indicated with black squares. The branch

support was calculated with 1000 ultrafast bootstrap replicates. (b) Structure results K from

2 to 5 , each vertical bar corresponds to the sample in the phylogenetic tree above Structure graphs. The height of the bar represents posterior probability of sample assignment to color

coded genetic groups. (c) PCA represents distinct genetic groups within the studied dataset.

Each dot indicates one individual. Percentages between brackets represent variance explained

by each principal component (PC). For isolates and locations abbreviations see Table S1. 


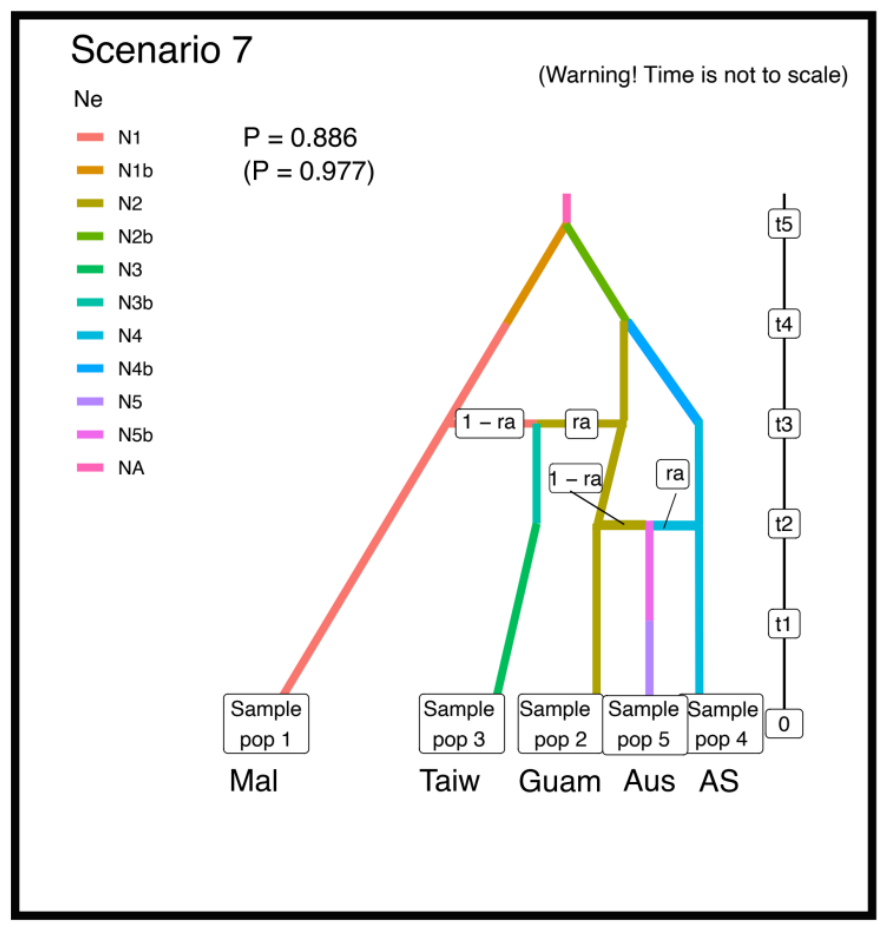

Figure 2. Winning hypothetical evolutionary scenario of the invasion history of Phellinus noxius in eastern Asia, the Pacific Islands, and Australia based on the results of five sequential DIYABC-RF analyses (see Supplementary materials for details about all tested scenarios in each analysis). P - posterior probability averaged across 5 independent runs for analysis 4 . Value between brackets is a posterior probability of scenarios group containing the winning scenario (see Methods for details). Mal - Malaysia, Taiw - Taiwan, Aus - Australia, and AS - American Samoa. 

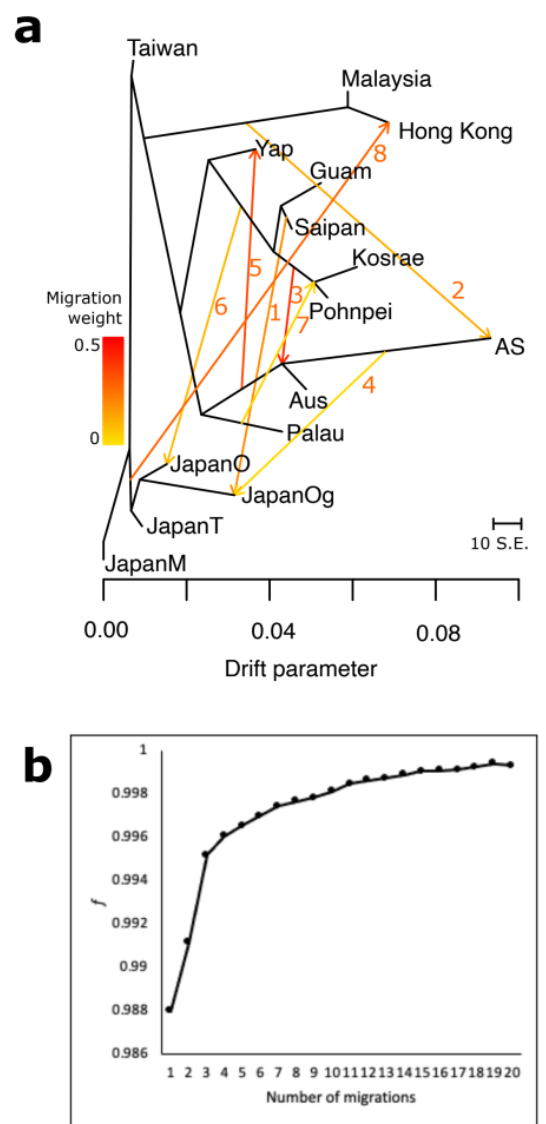

Figure 3. (a) Phylogenetic network inferred by Treemix of the relationships among Phellinus noxius populations with eight migration edges that explain $99.8 \%$ variance in the model. The migration edges are numbered from 1 to 8 reflecting the order in which they were added to the model (b) findex representing the fraction of the the model. (b) findex representing the fraction of the variance in the sample covariance matrix accounted for by the model covariance matrix as a function of the number of modelled migration events. For populations abbreviations see Table S1. 


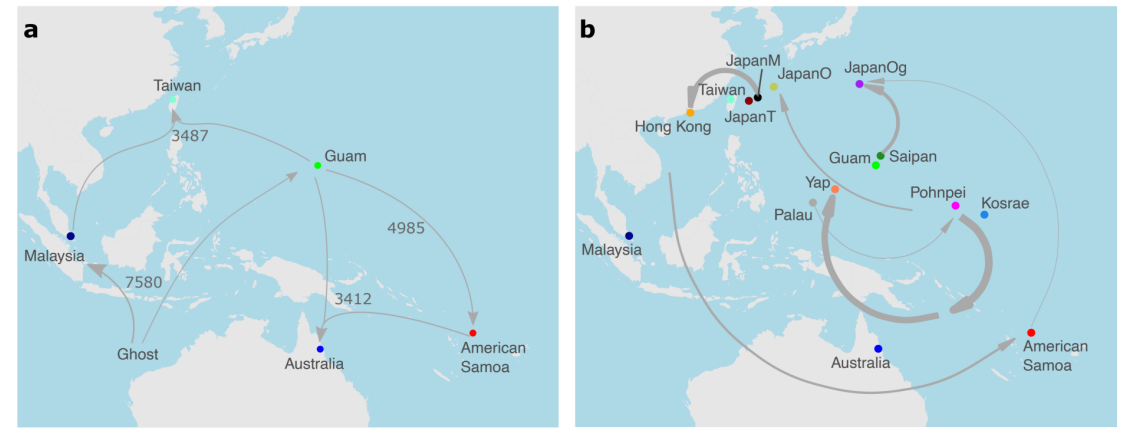

Figure 4. (a) Invasion routes of Phellinus noxius determined with DIYABC Random Forest analyses. The analysess were performed among populations
representing five genetic groups identified in this study. Numbers next to the lines represent number of generations. "Ghost" indicates ghost population with unknown location that gave origin to populations from Malaysia and Guam. (b) Migration among Phellinus noxius populations sampled from 15 locations detected unknown location that gave origin to populations from Malaysia and Guam. (b) Migration among Phellinus noxius populations sampled from 15 locations detected
with Treemix. The width of the lines reflects the percentage of ancestry (migration weight) in recipient from donor population (see Fig. 3 and main text). Migration to American Samoa (AS) traces back to the ancestral origins in Malaysia and/or Hong Kong. Migration to Yap traces back to the ancestral origins in Australia
and/or AS, migration to the ancestor of Australia and AS traces back to the ancestral origins in Pohnpei and Kosrae, and migration to Hong Kong traces back to the ancestral populations sampled from islands of Japan. 